\title{
RANKING OF TRIANGULAR TYPE-2 FUZZY SETS AND ITS APPLICATION IN MULTICRITERIA DECISION MAKING PROBLEM
}

\author{
Pushpinder Singh ${ }^{1}$, Manoj Kumar ${ }^{2}$, Shin Min Kang ${ }^{3}$ \\ ${ }^{1,2}$ Department of Mathematics \\ Lovely Professional University \\ Jalandhar - Delhi G.T. Road (NH-1) \\ Phagwara, Punjab 144411, INDIA \\ ${ }^{3}$ Department of Mathematics and RINS \\ Gyeongsang National University \\ Jinju, 52828, KOREA
}

\begin{abstract}
Type-2 fuzzy sets are the generalization of ordinary fuzzy sets which consider secondary membership function and primary membership function. In this paper a new approach is proposed for the ranking of triangular type-2 fuzzy sets by considering both secondary membership function and uncertainty in primary membership function. It is shown that the proposed approach satisfies reasonable properties for the ordering of fuzzy quantities. Comparison with existing method is also presented. For the illustration of the proposed approach some numerical examples are given. Application of proposed ranking function in multicriteria decision making problem is also presented.
\end{abstract}

AMS Subject Classification: $05 \mathrm{C} 72$

Key Words: fuzzy sets, type-2 fuzzy sets, ranking functions, multicriteria decision making problems

Received: July 22, 2016

Revised: $\quad$ August 20, 2016

Published: September 30, 2016 (c) 2016 Academic Publications, Ltd. url: www.acadpubl.eu 


\section{Introduction}

Zadeh [35] introduced the type-2 fuzzy set concept as an extension of an ordinary fuzzy set, that is, a type-1 fuzzy set. A type- 2 fuzzy set is a fuzzy set whose membership values are type- 1 fuzzy sets on $[0,1]$. The membership function of type-2 fuzzy sets provides an additional degree of freedom that makes it possible to directly model uncertainty. Type- 2 fuzzy sets are relatively hard to understand and clarify as compared to fuzzy sets. Type-2 fuzzy sets are three dimensional and can better improve certain kind of interface with increasing imprecision, uncertainty and fuzziness in information.

In order to rank fuzzy numbers, one fuzzy number needs to be evaluated and compared to others but this may not be easy. As known, the real numbers in $\mathbb{R}$ can be linearly ordered by $\geq$, however, fuzzy numbers cannot be done in such a way. Since fuzzy numbers are represented by possibility distributions, they can overlap with each other and it is difficult to determine clearly whether one fuzzy number is larger or smaller than the other. To the task of comparing fuzzy numbers, many authors proposed fuzzy ranking methods $[1,2,3,5,7,9$, $13,14,15,16,17,18,25,26,27,28]$.

Dubois and Prade [6], Hisdal [8], Mizumotto and Tanka [23, 24] and Yager [34] has investigated many useful results and properties of type-2 fuzzy sets. Type-2 fuzzy sets has gained more interest when, John [10], John and Czarnecki [12], Mendel [19], Mental and John [20] and Turksen [29, 30] initiated the research into type-2 fuzzy sets. Mitchell [22] considered the problem of ranking a set of type-2 fuzzy numbers from the statistical viewpoint and interpret each type- 2 fuzzy number as an ensemble of ordinary fuzzy numbers. Wu and Mendel [33] give a comprehensive study of ranking of interval type- 2 fuzzy sets and proposed the new ranking method based on the centroid of interval type- 2 fuzzy sets. On the basis of reasonable properties they concluded that Mitchell ranking method has some limitations. Also Wu and Mendel [33] satisfies four reasonable properties whereas Michell ranking method [22] satisfies only one.

$\mathrm{Wu}$ and Mendel [33] proposed the method for ranking of interval type-2 fuzzy sets. Also interval type-2 fuzzy sets are comparatively easy to understand and required less computation work.

In this paper, a new approach is proposed for the ranking of triangular type-2 fuzzy sets. In the proposed approach we are considering both centroid of secondary membership function and footprints of uncertainty as a union of primary membership function. It is shown that proposed method satisfies five reasonable properties for the ordering of fuzzy quantities, so it is more reasonable than the existing ranking method $[22,33]$. 
The rest of the paper is organized as follows: Section 2, introduce some basic definitions of type-2 fuzzy sets and a new representation of triangular type- 2 fuzzy sets. In Section 3 a new approach is proposed for the ranking of triangular type-2 fuzzy sets and some properties of centroid and footprint of uncertainty of triangular type-2 fuzzy are discussed. For the validation of proposed ranking approach, in Section 4 it is shown that the reasonable properties for the ordering of fuzzy qualities are satisfied and also the proposed approach is compared with some existing ranking approaches. Application of proposed ranking function in multicriteria decision making problem is discussed in Section 5. Finally, conclusions are drawn in Section 6 .

\section{Basic Definitions}

A type-2 fuzzy set was first proposed by Zadeh [35] as an extension of fuzzy sets. Many authors has applied type-2 fuzzy sets in different area, such as decision theory, signal processing, speech recognition, transport scheduling, pattern recognition, correlation coefficient, etc. A brief review of type-2 fuzzy sets is as follows:

Definition 2.1. ([35]) A type-2 fuzzy set is a set whose membership values are type-1 fuzzy sets on $[0,1]$.

Definition 2.2. ([20]) A type-2 fuzzy set, denoted by $\widetilde{A}$, is characterized by a type- 2 membership function $\xi_{\widetilde{A}}(x, u)$, where $x \in X$ and $\xi \in J_{x} \subseteq[0,1]$, that is, $\left.\widetilde{A}=\left\{(x, u), \xi_{\widetilde{A}}(x, u)\right) \mid \forall x \in X, \forall u \in J_{x} \subseteq[0,1]\right\}$ in which $0 \leq \xi_{\widetilde{A}}(x, u) \leq 1$.

$\widetilde{A}$ can also be expressed as $\widetilde{A}=\int_{x \in X} \int_{u \in J_{x}} \frac{\xi_{\widetilde{A}}(x, u)}{(x, u)}, J_{x} \subseteq[0,1]$, where $\iint$ denoted the union over all admissible $x$ and $u$. For a universe of discreet discourse, $\int$ is replaced by $\sum$.

Definition 2.3. ([20]) The domain of secondary membership function is called the primary membership of $x$. In the representation of $\widetilde{A}, J_{x}$ is the primary membership of $x$, where $J_{x} \subseteq[0,1], \forall x \in X$.

Definition 2.4. ([20]) Uncertainty in the primary membership of type-2 fuzzy sets, $\widetilde{A}$ consists of a bounded region that we call the footprint of uncertainty $(\mathrm{FOU})$. It is the union of all primary membership, that is, $\operatorname{FOU}(\widetilde{A})=$ $\bigcup J_{x}, \forall x \in X$. 


\subsection{Triangular Type-2 Fuzzy Sets}

In this section, we discuss why the notion of Triangular type-2 fuzzy set is needed and then, a new representation of triangular type- 2 fuzzy set is presented.

The computational complexity of general type-2 fuzzy sets is very large therefore, in the literature $[4,11,21,32]$ interval type-2 fuzzy sets was frequently used. The computations associated with interval type-2 fuzzy sets are very manageable, which make interval type-2 fuzzy sets quite practical. In interval type- 2 fuzzy sets that the secondary grades are equal to 1 means that the membership degree of the element $x_{i}$ in the universe $X$ is characterized by interval $\left[\underline{\xi}_{\widetilde{A}}(x), \bar{\xi}_{\widetilde{A}}(x)\right]$, as a subset of $[0,1]$. It is different from type-1 fuzzy sets, in which membership degrees are point values, any of the values in the subinterval can be assigned as the membership degree and each value has the same probability.

Now, we introduce the new representation of triangular type-2 fuzzy sets.

Definition 2.5. Let $X$ be the universe of discourse. A triangular type-2 fuzzy sets, denoted as $\widetilde{A}=\left\{(a, c, b ; \xi(c)),\left(a^{\prime}, c^{\prime}, b^{\prime} ; b\right)\right\}$, is characterized by a linear function $\xi_{\widetilde{A}}(u)$ as shown in Fig. 1. It is defined as follows:

$$
\xi_{\widetilde{A}}(u)= \begin{cases}0, & 0 \leq u<a, b \leq u<1 \\ \frac{\xi(c)(u-a)}{(c-a)}, & a \leq u<c \\ \frac{\xi(c)(u-b)}{(c-b)}, & c \leq u \leq b\end{cases}
$$

where $a<c<b$ and $\xi(c)$ is the maximum value of the secondary membership $\xi(u)$, that is, $\max (\xi(u))=\xi(c)$. $u$ is the domain of secondary membership function, called the primary membership function, is characteristic by a linear function $u_{\widetilde{A}}(x)$ as shown in Fig. 2 . It is defined as follows:

$$
u_{\widetilde{A}}(x)= \begin{cases}0, & 0 \leq x<a^{\prime}, b^{\prime} \leq x<1 \\ \frac{b\left(x-a^{\prime}\right)}{\left(c^{\prime}-a^{\prime}\right)}, & a^{\prime} \leq x<c^{\prime} \\ \frac{b\left(x-b^{\prime}\right)}{\left(c^{\prime}-b^{\prime}\right)}, & c^{\prime} \leq x \leq b^{\prime}\end{cases}
$$

where $0<a^{\prime}<c^{\prime}<b^{\prime}<1$ and $b$ is the maximum value of the primary membership $u$, that is, $\max (u)=b$. 

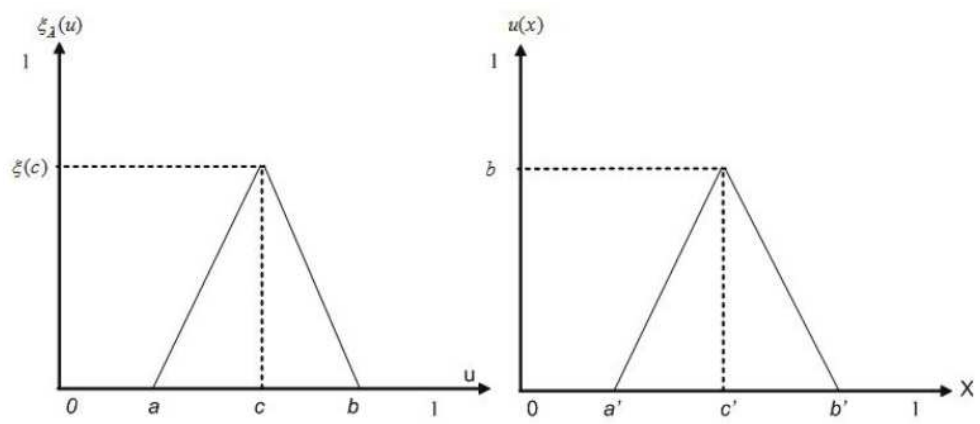

Fig. 1 The secondary membership function of $\tilde{A} \quad$ Fig. 2 The primary membership function of $\tilde{A}$

The triangular type-2 fuzzy set $\widetilde{A}_{i}=\left\{\left(a_{i}, c_{i}, b_{i} ; \xi\left(c_{i}\right)\right),\left(a_{i}^{\prime}, c_{i}^{\prime}, b_{i}^{\prime} ; b_{i}\right)\right\}$, together with primary and secondary membership function is shown in Fig. 3.

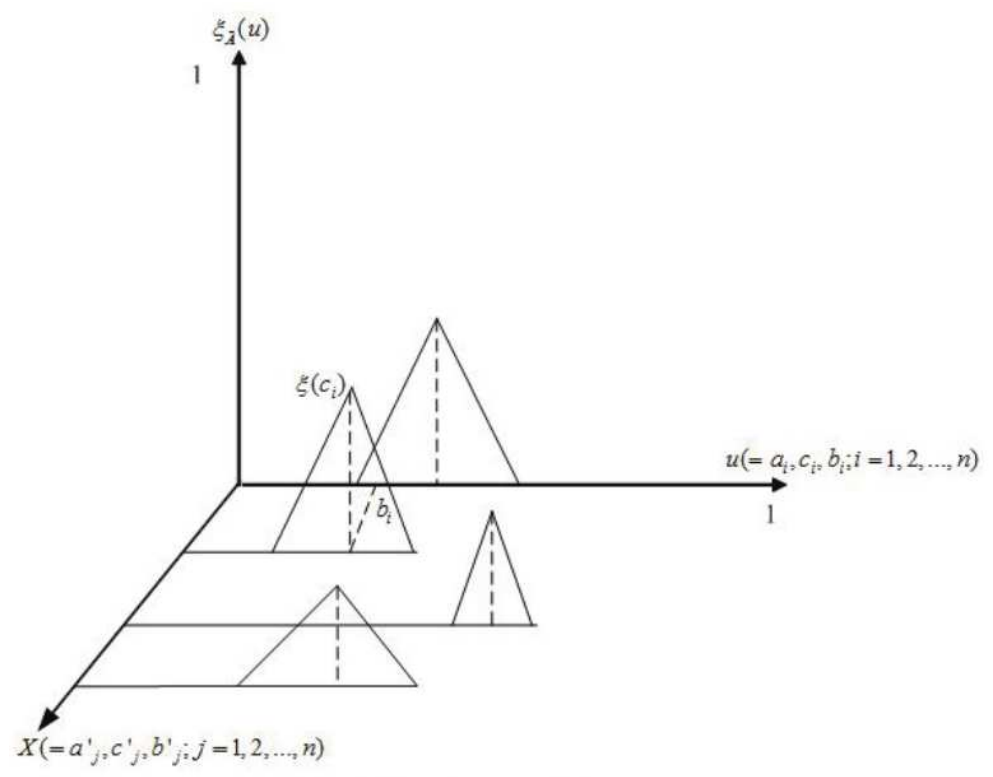

Fig. 3. Triangular type-2 fuzzy sets

\section{The Proposed Ranking Approach for Triangular Type-2 Fuzzy Sets}

In this section, we proposed a new method for the ranking of triangular type- 2 fuzzy sets. The proposed method consider both expectation value of centroid 
of the secondary membership function and uncertainty in primary membership function. Some properties of expectation value of centroid and uncertainty in primary membership function of triangular type-2 fuzzy sets are also discussed.

Definition 3.1. For any triangular type-2 fuzzy set $\widetilde{A}_{i}$, the expectation value of centroid of secondary membership function is defined as follows:

$$
M\left(\widetilde{A}_{i}\right)=\frac{\int_{a_{i}}^{b_{i}} u \xi_{\widetilde{A}_{i}}(u) d u}{\int_{a_{i}}^{b_{i}} \xi_{\widetilde{A}_{i}}(u) d u} .
$$

Since the secondary membership function of triangular type-2 fuzzy sets is given by

$$
\xi_{\widetilde{A_{i}}}(u)= \begin{cases}0, & 0 \leq u<a_{i}, b_{i} \leq u<1 \\ \frac{\xi\left(c_{i}\right)\left(u-a_{i}\right)}{\left(c_{i}-a_{i}\right)}, & a_{i} \leq u<c_{i} \\ \frac{\xi\left(c_{i}\right)\left(u-b_{i}\right)}{\left(c_{i}-b_{i}\right)}, & c_{i} \leq u \leq b_{i}\end{cases}
$$

Now,

$$
\int_{a_{i}}^{b_{i}} u \xi_{\widetilde{A}_{i}}(u) d u=\int_{a_{i}}^{c_{i}} u \xi_{\widetilde{A}_{i}}(u) d u+\int_{c_{i}}^{b_{i}} u \xi_{\widetilde{A}_{i}}(u) d u
$$

implies

$$
\int_{a_{i}}^{c_{i}} \frac{\xi\left(c_{i}\right)\left(u^{2}-a_{i} u\right)}{\left(c_{i}-a_{i}\right)} d u+\int_{c_{i}}^{b_{i}} \frac{\xi\left(c_{i}\right)\left(u^{2}-b_{i} u\right)}{\left(c_{i}-b_{i}\right)} d u
$$

and this implies

$$
\frac{\xi\left(c_{i}\right)}{6\left(c_{i}-a_{i}\right)\left(c_{i}-b_{i}\right)}\left\{c_{i}^{3}\left(b_{i}-a_{i}\right)+c_{i}\left(b_{i}^{3}-a_{i}^{3}\right)-a_{i} b_{i}\left(a_{i}^{2}+b_{i}^{2}\right)\right\} .
$$

Hence

$$
\begin{aligned}
& \int_{a_{i}}^{b_{i}} u \xi_{\widetilde{A}_{i}}(u) d u \\
& =\frac{\xi\left(c_{i}\right)}{6\left(c_{i}-a_{i}\right)\left(c_{i}-b_{i}\right)}\left\{c_{i}^{3}\left(b_{i}-a_{i}\right)+c_{i}\left(b_{i}^{3}-a_{i}^{3}\right)-a_{i} b_{i}\left(a_{i}^{2}-b_{i}^{2}\right)\right\} .
\end{aligned}
$$

Again,

$$
\int_{a_{i}}^{b_{i}} \xi_{\widetilde{A}_{i}}(u) d u=\int_{a_{i}}^{c_{i}} \xi_{\widetilde{A}_{i}}(u) d u+\int_{c_{i}}^{b_{i}} \xi_{\widetilde{A}_{i}}(u) d u
$$

implies

$$
\int_{a_{i}}^{c_{i}} \frac{\xi\left(c_{i}\right)\left(u-a_{i}\right)}{\left(c_{i}-a_{i}\right)} d u+\int_{c_{i}}^{b_{i}} \frac{\xi\left(c_{i}\right)\left(u-b_{i}\right)}{\left(c_{i}-b_{i}\right)} d u
$$


and this implies

$$
\frac{\xi\left(c_{i}\right)}{2\left(c_{i}-a_{i}\right)\left(c_{i}-b_{i}\right)}\left\{c_{i}^{2}\left(b_{i}-a_{i}\right)+c_{i}\left(b_{i}^{2}-a_{i}^{2}\right)+a_{i} b_{i}\left(a_{i}-b_{i}\right)\right\}
$$

Hence

$$
\begin{aligned}
& \int_{a_{i}}^{b_{i}} u \xi_{\widetilde{A}_{i}}(u) d u \\
& =\frac{\xi\left(c_{i}\right)}{2\left(c_{i}-a_{i}\right)\left(c_{i}-b_{i}\right)}\left\{c_{i}^{2}\left(b_{i}-a_{i}\right)+c_{i}\left(b_{i}^{2}-a_{i}^{2}\right)+a_{i} b_{i}\left(a_{i}-b_{i}\right)\right\}
\end{aligned}
$$

On substituting Eq.(3.2) and Eq.(3.3) in (3.1) we get

$$
M\left(\widetilde{A}_{i}\right)=\frac{1}{3} \frac{c_{i}^{3}\left(b_{i}-a_{i}\right)+c_{i}\left(b_{i}^{3}-a_{i}^{3}\right)-a_{i} b_{i}\left(a_{i}^{2}-b_{i}^{2}\right)}{c_{i}^{2}\left(b_{i}-a_{i}\right)+c_{i}\left(b_{i}^{2}-a_{i}^{2}\right)+a_{i} b_{i}\left(a_{i}-b_{i}\right)} .
$$

Definition 3.2. According to Mendel and John [20] the uncertainty in the primary membership of type- 2 fuzzy sets, $\widetilde{A}_{i}$ consists of a bounded region that we call the footprint of uncertainty $(\mathrm{FOU})$. Consider the primary membership function of triangular type-2 fuzzy sets $\widetilde{A}_{i}$ :

$$
u_{\widetilde{A}_{i}}(x)= \begin{cases}0, & 0 \leq x<a_{i}^{\prime}, b_{i}^{\prime} \leq x<1, \\ \frac{b_{i}\left(x-a_{i}^{\prime}\right)}{\left(c_{i}^{\prime}-a_{i}^{\prime}\right)}, & a_{i}^{\prime} \leq x<c_{i}^{\prime}, \\ \frac{b_{i}\left(x-b_{i}^{\prime}\right)}{\left(c_{i}^{\prime}-b_{i}^{\prime}\right)}, & c_{i}^{\prime} \leq x \leq b_{i}^{\prime} .\end{cases}
$$

The uncertainty in primary membership function (that is, FOU) of a triangular type-2 fuzzy set can be obtained by calculating the area under primary membership function, thus FOU can be obtained by using integral value of primary membership function. Assume that $\varphi_{\widetilde{A}_{i}}^{L}=\frac{b_{i}\left(x-a_{i}^{\prime}\right)}{\left(c^{\prime}-a_{i}^{\prime}\right)}$ is the left primary membership function and $\varphi_{\widetilde{A}_{i}}^{R}=\frac{b_{i}\left(x-b_{i}^{\prime}\right)}{\left(c_{i}^{\prime}-b_{i}^{\prime}\right)}$ is the right primary membership function function of triangular type-2 two fuzzy set. Suppose that $\varsigma_{\widetilde{A}_{i}}^{L}$ and $\varsigma_{\widetilde{A}_{i}}^{R}$ are the inverse functions of $\varphi_{\widetilde{A}_{i}}^{L}$ and $\varphi_{\widetilde{A}_{i}}^{R}$ respectively, as shown in Fig. 4. 


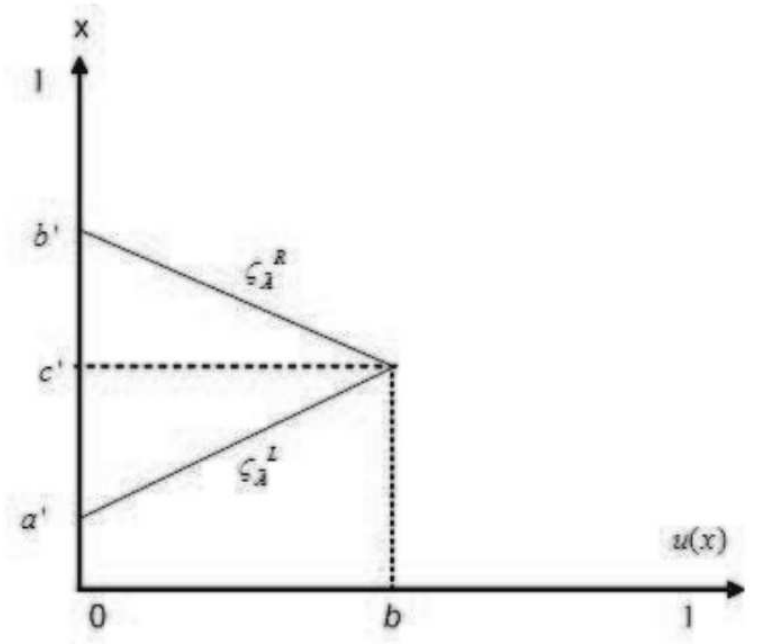

Fig, 4 Inverse image of the primary membership function

Therefore the area under primary membership function, or footprint of uncertainty is given by the equation $\int_{0}^{b_{i}} \varsigma_{\widetilde{A}_{i}}^{L}(y) d y+\int_{0}^{b_{i}} \varsigma_{\widetilde{A}_{i}}^{R}(y) d y$.

On solving above equation, we get

$$
\operatorname{FOU}\left(\widetilde{A}_{i}\right)=\frac{b_{i}\left(a_{i}^{\prime}+2 c_{i}^{\prime}+b_{i}^{\prime}\right)}{2} .
$$

To compute the ranking function for triangular type-2 fuzzy sets, we consider both FOU of the primary membership function and expectation value of the centroid of secondary membership function. From Definitions 3.1 and 3.2, we can propose ranking function for triangular type- 2 fuzzy set $\widetilde{A}_{i}$ as follows:

$$
\Re\left(\widetilde{A}_{i}\right)=\frac{1}{2}\left\{M\left(\widetilde{A}_{i}\right)+F O U\left(\widetilde{A}_{i}\right)\right\}
$$

From Eqs.(3.4) and (3.5) we get

$$
\Re\left(\widetilde{A}_{i}\right)=\frac{1}{2}\left\{\frac{1}{3} \frac{c_{i}^{3}\left(b_{i}-a_{i}\right)+c_{i}\left(b_{i}^{3}-a_{i}^{3}\right)-a_{i} b_{i}\left(a_{i}^{2}-b_{i}^{2}\right)}{c_{i}^{2}\left(b_{i}-a_{i}\right)+c_{i}\left(b_{i}^{2}-a_{i}^{2}\right)+a_{i} b_{i}\left(a_{i}-b_{i}\right)}+\frac{b_{i}\left(a_{i}^{\prime}+2 b_{i}^{\prime}+c_{i}^{\prime}\right)}{2}\right\} .
$$

\subsection{Algorithms}

Now, we will propose algorithms for the comparison of triangular type-2 fuzzy sets. 
Consider $n$ type- 2 fuzzy sets $\widetilde{A}_{i}, i=1,2, \ldots, n$, each with a triangular membership function. The proposed method performs pairwise comparison on the $n$ triangular type-2 fuzzy sets. For each pair of fuzzy sets, say $\widetilde{A}_{1}$ and $\widetilde{A}_{2}$, the pair wise comparison is proceeds as follows:

Let $\widetilde{A}_{1}=\left\{\left(a_{1}, c_{1}, b_{1} ; \xi\left(c_{1}\right)\right),\left(a_{1}^{\prime}, c_{1}^{\prime}, b_{1}^{\prime} ; b_{1}\right)\right\}$ and $\widetilde{A}_{2}=\left\{\left(a_{2}, c_{2}, b_{2} ; \xi\left(c_{2}\right)\right)\right.$, $\left.\left(a_{2}^{\prime}, c_{2}^{\prime}, b_{2}^{\prime} ; b_{2}\right)\right\}$ be two type-2 fuzzy sets with triangular secondary and primary memberships

$$
\begin{aligned}
& \xi_{\widetilde{A}_{1}}(u)= \begin{cases}0, & 0 \leq u<a_{1}, b_{1} \leq u<1, \\
\frac{\xi\left(c_{1}\right)\left(u-a_{1}\right)}{\left(c-a_{1}\right)}, & a_{1} \leq u<c_{1}, \\
\frac{\xi\left(c_{1}\right)\left(u-b_{1}\right)}{\left(c_{1}-b_{1}\right)}, & c_{1} \leq u \leq b_{1},\end{cases} \\
& \xi_{\widetilde{A}_{2}}(u)= \begin{cases}0, & 0 \leq u<a_{2}, b_{2} \leq u<1, \\
\frac{\xi\left(c_{2}\right)\left(u-a_{2}\right)}{\left(c-a_{2}\right)}, & a_{2} \leq u<c_{2}, \\
\frac{\xi\left(c_{2}\right)\left(u-b_{2}\right)}{\left(c_{1}-b_{2}\right)}, & c_{2} \leq u \leq b_{2},\end{cases}
\end{aligned}
$$

and

$$
\begin{aligned}
& u_{\widetilde{A}_{1}}(x)= \begin{cases}0, & 0 \leq x<a_{1}^{\prime}, b_{1}^{\prime} \leq x<1, \\
\frac{b_{1}\left(x-a_{1}^{\prime}\right)}{\left(c_{1}^{\prime}-a_{1}^{\prime}\right)}, & a_{1}^{\prime} \leq x<c_{1}^{\prime}, \\
\frac{b_{1}\left(x-b_{1}^{\prime}\right)}{\left(c_{1}^{\prime}-b_{1}^{\prime}\right)}, & c_{1}^{\prime} \leq x \leq b_{1}^{\prime},\end{cases} \\
& u_{\widetilde{A}_{2}}(x)= \begin{cases}0, & 0 \leq x<a_{2}^{\prime}, b_{2}^{\prime} \leq x<1, \\
\frac{b_{2}\left(x-a_{2}^{\prime}\right)}{\left(c_{2}^{\prime}-a_{2}^{\prime}\right)}, & a_{2}^{\prime} \leq x<c_{2}^{\prime}, \\
\frac{b_{2}\left(x-b_{2}^{2}\right)}{\left(c_{2}^{\prime}-b_{2}^{\prime}\right)}, & c_{2}^{\prime} \leq x \leq b_{2}^{\prime},\end{cases}
\end{aligned}
$$

respectively.

Use the following steps to compare $\widetilde{A}_{1}$ and $\widetilde{A}_{2}$ :

STEP 1: Standardize each triangular type-2 fuzzy sets by transforming $\widetilde{A}_{1}, \widetilde{A}_{2}$ into $\widetilde{A}_{1}^{*}, \widetilde{A}_{2}^{*}$, where $\widetilde{A}_{1}^{*}=\left\{\left(a_{1}, c_{1}, b_{1} ; \xi(c)\right),\left(a_{1}^{\prime}, c_{1}^{\prime}, b_{1}^{\prime} ; b\right)\right\}$ and $\widetilde{A}_{2}^{*}=$ $\left\{\left(a_{2}, c_{2}, b_{2} ; \xi(c)\right),\left(a_{2}^{\prime}, c_{2}^{\prime}, b_{2}^{\prime} ; b_{2}\right)\right\}$.

The secondary and primary membership function of $\widetilde{A}_{1}^{*}$ and $\widetilde{A}_{2}^{*}$ are given as:

$$
\xi_{\widetilde{A}_{1}^{*}}(u)= \begin{cases}0, & 0 \leq u<a_{1}, b_{1} \leq u<1 \\ \frac{\xi(c)\left(u-a_{1}\right)}{\left(c-a_{1}\right)}, & a_{1} \leq u<c_{1} \\ \frac{\xi(c)\left(u-b_{1}\right)}{\left(c_{1}-b_{1}\right)}, & c_{1} \leq u \leq b_{1}\end{cases}
$$




$$
\xi_{\widetilde{A}_{2}^{*}}(u)= \begin{cases}0, & 0 \leq u<a_{2}, b_{2} \leq u<1, \\ \frac{\xi(c)\left(u-a_{2}\right)}{\left(c-a_{2}\right)}, & a_{2} \leq u<c_{2}, \\ \frac{\xi(c)\left(u-b_{2}\right)}{\left(c_{1}-b_{2}\right)}, & c_{2} \leq u \leq b_{2},\end{cases}
$$

and

$$
\begin{gathered}
u_{\widetilde{A}_{1}^{*}}(x)= \begin{cases}0, & 0 \leq x<a_{1}^{\prime}, b_{1}^{\prime} \leq x<1, \\
\frac{b\left(x-a_{1}^{\prime}\right)}{\left(c_{1}^{\prime}-a_{1}^{\prime}\right)}, & a_{1}^{\prime} \leq x<c_{1}^{\prime}, \\
\frac{b\left(x-b_{1}\right)}{\left(c_{1}^{\prime}-b_{1}^{\prime}\right)}, & c_{1}^{\prime} \leq x \leq b_{1}^{\prime},\end{cases} \\
u_{\widetilde{A}_{2}^{*}}(x)= \begin{cases}0, & 0 \leq x<a_{2}^{\prime}, b_{2}^{\prime} \leq x<1, \\
\frac{b\left(x-a_{2}^{\prime}\right)}{\left(c_{2}^{\prime}-a_{2}^{\prime}\right)}, & a_{2}^{\prime} \leq x<c_{2}^{\prime}, \\
\frac{b\left(x-b_{2}^{2}\right)}{\left(c_{2}^{\prime}-b_{2}^{\prime}\right)}, & c_{2}^{\prime} \leq x \leq b_{2}^{\prime},\end{cases}
\end{gathered}
$$

respectively, where $\xi(c)=\min \left(\xi\left(c_{1}\right), \xi\left(c_{2}\right)\right)$ and $b=\min \left(b_{1}, b_{2}\right)$.

STEP 2: Using Eq.(3.4) and Eq.(3.5) calculate $M_{1}\left(\widetilde{A}_{1}^{*}\right), M_{2}\left(\widetilde{A}_{2}^{*}\right)$ and FOU $\left(\widetilde{A}_{1}^{*}\right)$, $\operatorname{FOU}\left(\widetilde{A}_{2}^{*}\right)$.

SteP 3: Using Eq.(3.6) calculate $\Re\left(\widetilde{A}_{1}^{*}\right)$ and $\Re\left(\widetilde{A}_{2}^{*}\right)$. Then, $\widetilde{A}_{1}$ and $\widetilde{A}_{2}$ can be compared as follows:

Case 1. $\widetilde{A}_{1} \succeq \widetilde{A}_{2}$ if $\Re\left(\widetilde{A}_{1}^{*}\right) \succeq \Re\left(\widetilde{A}_{2}^{*}\right)$.

Case 2. $\widetilde{A}_{1} \preceq \widetilde{A}_{2}$ if $\Re\left(\widetilde{A}_{1}^{*}\right) \preceq \Re\left(\widetilde{A}_{2}^{*}\right)$.

Case 3. $\widetilde{A}_{1} \sim \widetilde{A}_{2}$ if $\Re\left(\widetilde{A}_{1}^{*}\right) \sim \Re\left(\widetilde{A}_{2}^{*}\right)$.

Definition 3.3. Two triangular type-2 fuzzy sets $\widetilde{A}_{1}$ and $\widetilde{A}_{2}$ are said to have the same shape if their secondary and primary membership function satisfies the following relation:

$$
\xi_{\widetilde{A}_{2}}(v)=\xi_{\widetilde{A}_{1}}(u) \quad \text { and } \quad v_{\widetilde{A}_{1}}(x)=u_{\widetilde{A}_{2}}(x), \quad \forall v=a u+b,
$$

where $a \neq 0$ and $a, b \in R$.

Theorem 3.4. If triangular type-2 fuzzy sets $\widetilde{A}_{1}$ and $\widetilde{A}_{2}$ with secondary membership functions $\xi_{\widetilde{A}_{2}}(u)$ and $\xi_{\widetilde{A}_{1}}(u)$, respectively, satisfies the relation $\xi_{\widetilde{A}_{2}}(v)=\xi_{\widetilde{A}_{1}}(u)$, where $v=a u+b$, then $M\left(\widetilde{A}_{2}\right)=a M\left(\widetilde{A}_{1}\right)+b$.

Proof. Let $v=a u+b$.

$$
\begin{array}{ll}
\int_{a_{1}}^{b_{1}} \xi_{\widetilde{A}_{2}}(v) d v=\int_{a_{1}}^{b_{1}} a \xi_{\widetilde{A}_{1}}(u) d u=|a| \int_{a_{1}}^{b_{1}} \xi_{\widetilde{A}_{1}}(u) d u & \text { if } a>0, \\
\int_{a_{1}}^{b_{1}} \xi_{\widetilde{A}_{2}}(v) d v=\int_{a_{1}}^{b_{1}} a \xi_{\widetilde{A}_{1}}(u) d u=|a| \int_{a_{1}}^{b_{1}} \xi_{\widetilde{A}_{1}}(u) d u & \text { if } a<0 .
\end{array}
$$


Thus, we have

$$
\int_{a_{1}}^{b_{1}} \xi_{\widetilde{A}_{2}}(v) d v=|a| \int_{a_{1}}^{b_{1}} \xi_{\widetilde{A}_{1}}(u) d u
$$

Similarly, we obtain

$$
\int_{a_{1}}^{b_{1}} v \xi_{\widetilde{A}_{2}}(v) d v=|a| \int_{a_{1}}^{b_{1}}(a u+b) \xi_{\widetilde{A}_{1}}(u) d u
$$

Therefore, we get

$$
\begin{aligned}
M\left(\widetilde{A}_{2}\right) & =\frac{\int_{a_{1}}^{b_{1}} v \xi_{\widetilde{A}_{2}}(v) d v}{\int_{a_{1}}^{b_{1}} \xi_{\widetilde{A}_{2}}(v) d v}=\frac{\int_{a_{1}}^{b_{1}}(a u+b) \xi_{\widetilde{A}_{1}}(u) d u}{\int_{a_{1}}^{b_{1}} \xi_{\widetilde{A}_{1}}(u) d u} \\
& =a \frac{\int_{a_{1}}^{b_{1}}(u) \xi_{\widetilde{A}_{1}}(u) d u}{\int_{a_{1}}^{b_{1}} \xi_{\widetilde{A}_{1}}(u) d u}+b=a M\left(\widetilde{A}_{2}\right)+b .
\end{aligned}
$$

Hence, $M\left(\widetilde{A}_{2}\right)=a M\left(\widetilde{A}_{1}\right)+b$.

Theorem 3.5. If $\widetilde{A}_{1}$ and $\widetilde{A}_{2}$ are triangular type-2 fuzzy sets with its secondary membership function satisfying the condition $\xi_{\widetilde{A}_{2}}(u)=\xi(c) \xi_{\widetilde{A}_{1}}(u)$, where $\widetilde{A}_{1}$ is a assumed to be a triangular type-2 fuzzy sets with degree of secondary membership unity and $0<\xi(c)<1$, then $M\left(\widetilde{A}_{2}\right)=M\left(\widetilde{A}_{1}\right)$.

Proof.

$$
\begin{aligned}
M\left(\widetilde{A}_{2}\right) & =\frac{\int_{a_{1}}^{b_{1}} v \xi_{\widetilde{A}_{2}}(v) d v}{\int_{a_{1}}^{b_{1}} \xi_{\widetilde{A}_{2}}(v) d v}=\frac{\xi(c) \int_{a_{1}}^{b_{1}} v \xi_{\widetilde{A}_{1}}(v) d v}{\xi(c) \int_{a_{1}}^{b_{1}} \xi_{\widetilde{A}_{1}}(v) d v} \\
& =M\left(\widetilde{A}_{1}\right) .
\end{aligned}
$$

Hence, $M\left(\widetilde{A}_{2}\right)=M\left(\widetilde{A}_{1}\right)$.

Theorem 3.6. Let $\widetilde{A}_{1}$ be triangular type-2 fuzzy set and $-\widetilde{A}_{1}$ be the image of $\widetilde{A}_{1}$. Then $M\left(\widetilde{A}_{1}\right)=-M\left(-\widetilde{A}_{1}\right)$.

Proof. From Eq.(3.4), we have

$$
M\left(\widetilde{A}_{1}\right)=\frac{1}{3} \frac{c_{1}^{3}\left(b_{1}-a_{1}\right)+c_{1}\left(b_{1}^{3}-a_{1}^{3}\right)-a_{1} b_{1}\left(a_{1}^{2}-b_{1}^{2}\right)}{c_{1}^{2}\left(b_{1}-a_{1}\right)+c_{1}\left(b_{1}^{2}-a_{1}^{2}\right)+a_{1} b_{1}\left(a_{1}-b_{1}\right)} .
$$

On replacing $a_{1}, c_{1}, b_{1}$ with $-b_{1},-c_{1},-a_{1}$, respectively, in above equation we get

$$
M\left(\widetilde{A}_{1}\right)=\frac{1}{3} \frac{-c_{1}^{3}\left(-a_{1}+b_{1}\right)-c_{1}\left(-a_{1}^{3}+b_{1}^{3}\right)-b_{1} a_{1}\left(b_{1}^{2}-a_{1}^{2}\right)}{-c_{1}^{2}\left(-a_{1}+b_{1}\right)-c_{i}\left(-a_{1}^{2}+b_{1}^{2}\right)+b_{1} a_{1}\left(-b_{1}+a_{1}\right)} .
$$


Hence we have

$$
M\left(\widetilde{A}_{1}\right)=-M\left(-\widetilde{A}_{1}\right)
$$

Theorem 3.7. If $\widetilde{A}_{1}$ and $\widetilde{A}_{2}$ are two triangular type-2 fuzzy sets with same degree of primary membership function, $\underset{\widetilde{F} O U}{ }\left(\widetilde{A}_{1}\right)$ and $\operatorname{FOU}\left(\widetilde{A}_{2}\right)$ are their footprints of uncertainty, then $\operatorname{FOU}\left(\widetilde{A}_{1}+\widetilde{A}_{2}\right)=\operatorname{FOU}\left(\widetilde{A}_{1}\right)+\operatorname{FOU}\left(\widetilde{A}_{2}\right)$.

Proof. Let $\widetilde{A}_{1}$ and $\widetilde{A}_{2}$ be two triangular type-2 fuzzy sets with primary membership function

$$
u_{\widetilde{A}_{1}}(x)= \begin{cases}0, & 0 \leq x<a_{1}^{\prime}, b_{1}^{\prime} \leq x<1, \\ \frac{b\left(x-a_{1}^{\prime}\right)}{\left(c_{1}^{\prime}-a_{1}^{\prime}\right)}, & a_{1}^{\prime} \leq x<c_{1}^{\prime}, \\ \frac{b\left(x-b_{1}^{1}\right)}{\left(c_{1}^{\prime}-b_{1}^{\prime}\right)}, & c_{1}^{\prime} \leq x \leq b_{1}^{\prime},\end{cases}
$$

and

$$
u(x)_{\widetilde{A}_{2}}= \begin{cases}0, & 0 \leq x<a_{2}^{\prime}, b_{2}^{\prime} \leq x<1, \\ \frac{b\left(x-a_{2}^{\prime}\right)}{\left(c_{2}^{\prime}-a_{2}^{\prime}\right)}, & a_{2}^{\prime} \leq x<c_{2}^{\prime}, \\ \frac{b\left(x-b_{2}^{\prime}\right)}{\left(c_{2}^{\prime}-b_{2}^{\prime}\right)}, & c_{2}^{\prime} \leq x \leq b_{2}^{\prime} .\end{cases}
$$

Since primary membership functions are type- 1 , therefore $\widetilde{A}_{1}+\widetilde{A}_{2}=\left(a_{1}^{\prime}+\right.$ $\left.a_{2}^{\prime}, b_{1}^{\prime}+b_{2}^{\prime}, c_{1}^{\prime}+c_{2}^{\prime} ; b\right)[13]$.

Now using Eq.(3.5) we have

$$
\begin{aligned}
\operatorname{FOU}\left(\widetilde{A}_{1}+\widetilde{A}_{2}\right) & =\frac{b\left(a_{1}^{\prime}+a_{2}^{\prime}+2\left(c_{1}^{\prime}+c_{2}^{\prime}\right)+b_{1}^{\prime}+b_{2}^{\prime}\right)}{2} \\
& =\frac{b\left(a_{1}^{\prime}+2 c_{1}^{\prime}+b_{1}^{\prime}\right)}{2}+\frac{b\left(a_{2}^{\prime}+2 c_{2}^{\prime}+b_{2}^{\prime}\right)}{2} \\
& =F O U\left(\widetilde{A}_{1}\right)+F O U\left(\widetilde{A}_{2}\right) .
\end{aligned}
$$

Hence $\operatorname{FOU}\left(\widetilde{A}_{1}+\widetilde{A}_{2}\right)=\operatorname{FOU}\left(\widetilde{A}_{1}\right)+\operatorname{FOU}\left(\widetilde{A}_{2}\right)$.

\section{Examples, Reasonable Properties for the Ordering of Fuzzy Quantities and Comparative Study}

In this section some illustrative examples for the comparison of triangular type-2 fuzzy sets are presented and it is shown that proposed ranking approach satisfies the reasonable properties for the ordering of fuzzy quantities. Also comparison with some existing ranking approaches has been given. 
Example 4.1. Let $\widetilde{A}_{1}=\{(0.1,0.4,0.5 ; 0.3),(0.2,0.3,0.4 ; 0.4)\}$ and $\widetilde{A}_{2}=$ $\{(0.3,0.4,0.7, ; 0.2),(0.4,0.5,0.6 ; 0.6)\}$ be triangular type-2 fuzzy sets. Use the following steps to compare $\widetilde{A}_{1}$ and $\widetilde{A}_{2}$ :

SteP 1: Transform $\widetilde{A}_{1}, \widetilde{A}_{2}$ into $\widetilde{A}_{1}^{*}$ and $\widetilde{A}_{2}^{*}$, where $\widetilde{A}_{1}^{*}=\{(0.1,0.4,0.5 ; 0.2)$, $(0.2,0.3,0.4 ; 0.4)\}$ and $\widetilde{A}_{2}^{*}=\{(0.3,0.4,0.7, ; 0.2),(0.4,0.5,0.6 ; 0.4)\}$.

STEP 2: Using Eq.(3.4) and (3.5), we get $M\left(\widetilde{A}_{1}^{*}\right)=0.2076, M\left(\widetilde{A}_{2}^{*}\right)=0.5619$ and $\operatorname{FOU}\left(\widetilde{A}_{1}^{*}\right)=0.24, F O U\left(\widetilde{A}_{2}^{*}\right)=0.6$.

SteP 3: Using Eq.(3.6), we get $\Re\left(\widetilde{A}_{1}^{*}\right)=0.2238$ and $\Re\left(\widetilde{A}_{2}^{*}\right)=0.5809$. Since $\Re\left(\widetilde{A}_{1}^{*}\right)<\Re\left(\widetilde{A}_{2}^{*}\right), \widetilde{A}_{1} \prec \widetilde{A}_{2}$.

Example 4.2. Let $\widetilde{A}_{1}=\{(0.3,0.5,0.8 ; 0.7),(0.4,0.5,0.6 ; 0.6)\}$ and $\widetilde{A}_{2}=$ $\{(0.2,0.3,0.6, ; 0.5),(0.3,0.4,0.5 ; 0.4)\}$ be triangular type-2 fuzzy sets. Use the following steps to compare $\widetilde{A}_{1}$ and $\widetilde{A}_{2}$ :

SteP 1: Transform $\widetilde{A}_{1}, \widetilde{A}_{2}$ into $\widetilde{A}_{1}^{*}$ and $\widetilde{A}_{2}^{*}$, where $\widetilde{A}_{1}^{*}=\{(0.3,0.5,0.8 ; 0.5)$, $(0.4,0.5,0.6 ; 0.4)\}$ and $\widetilde{A}_{2}^{*}=\{(0.2,0.3,0.6, ; 0.5),(0.3,0.4,0.5 ; 0.4)\}$ are type- 2 fuzzy sets.

SteP 2: Using Eqs.(3.4) and (3.5), we get $M_{1}\left(\widetilde{A}_{1}^{*}\right)=0.5202, M_{2}\left(\widetilde{A}_{2}^{*}\right)=$ 0.4428 and $\operatorname{FOU}\left(\widetilde{A}_{1}^{*}\right)=0.6, \operatorname{FOU}\left(\widetilde{A}_{2}^{*}\right)=0.32$.

SteP 3: Using Eq.(3.6), we get $\Re\left(\widetilde{A}_{1}^{*}\right)=0.5601$ and $\Re\left(\widetilde{A}_{2}^{*}\right)=0.4428$. Since $\Re\left(\widetilde{A}_{1}^{*}\right)>\Re\left(\widetilde{A}_{2}^{*}\right), \widetilde{A}_{1} \succ \widetilde{A}_{2}$.

Wang and Kerre [31] proposed some axioms as a reasonable properties of ordering fuzzy quantities for the ranking approach $\Re$. These properties are:

$(P 1)$ For an arbitrary subset $S$ of $F_{2}$ and $\widetilde{A} \in S, \widetilde{A} \succeq \widetilde{A}$ by $\Re$ on $S$, where $F_{2}$ is a set of triangular type-2 fuzzy sets.

$(P 2)$ For an arbitrary subset $S$ of $F_{2}$ and $\widetilde{A}, \widetilde{B} \in S$, if $\widetilde{A} \succeq \widetilde{B}$ and $\widetilde{B} \succeq \widetilde{A}$ by $\Re$ on $S$, then $\widetilde{A} \sim \widetilde{B}$ by $\Re$ on $S$.

$(P 3)$ For an arbitrary subset $S$ of $F_{2}$ and $\widetilde{A}, \widetilde{B}, \widetilde{C} \in S$, if $\widetilde{A} \succeq \widetilde{B}$ and $\widetilde{B} \succeq \widetilde{C}$ by $\Re$ on $S$, then $\widetilde{A} \sim \widetilde{C}$ by $\Re$ on $S$.

(P4) If $\widetilde{A} \cap \widetilde{B}=\emptyset$ and $\widetilde{A}$ is on the right of $\widetilde{B}$, then $\widetilde{A} \succeq \widetilde{B}$.

$(P 5)$ Let $S$ and $S^{\prime}$ be two arbitrary finite sets of triangular type-2 fuzzy sets in which proposed ranking function can be applied, and $\widetilde{A}, \widetilde{B} \in S \cap S^{\prime}$. Then we obtain the ranking order $\widetilde{A} \succeq \widetilde{B}$ by $\Re$ in $S$ if and only if $\widetilde{A} \succeq \widetilde{B}$ by $\Re$ in $S^{\prime}$.

(P6) If $\widetilde{A} \succeq \widetilde{B}$ by $\Re$, then $\widetilde{\widetilde{A}}+\widetilde{C} \succeq \widetilde{B}+\widetilde{C}$ by $\Re$ when $\widetilde{C} \neq 0$.

(P7) Let $\widetilde{\widetilde{A}}, \widetilde{B}, \widetilde{A} \widetilde{C}, \widetilde{B} \widetilde{C}$ be the elements of $S$ and $\widetilde{C} \geq 0$. $\widetilde{A} \succeq \widetilde{B}$ by $\Re$ implies $\widetilde{A} \widetilde{C} \succeq \widetilde{B} \widetilde{C}$ by $\Re$.

Now, we prove that proposed ranking function satisfies some of the reasonable properties. 
Proposition 4.3. For an arbitrary subset $S$ of $F_{2}$ and $\widetilde{A} \in S, \widetilde{A} \succeq \widetilde{A}$ by $\Re$ on $S$.

Proof. Since $\widetilde{A} \in S, \widetilde{A}, \widetilde{A} \in S$ and $\Re(\widetilde{A}) \geq \Re(\widetilde{A})$, which implies that $\widetilde{A} \succeq \widetilde{A}$ by proposed ranking function.

Proposition 4.4. For an arbitrary subset $S$ of $F_{2}$ and $\widetilde{A}, \widetilde{B} \in S$, if $\widetilde{A} \succeq \widetilde{B}$ and $\widetilde{B} \succeq \widetilde{A}$ by $\Re$ on $S$, then $\widetilde{A} \sim \widetilde{B}$ by $\Re$ on $S$.

Proof. By proposed ranking function, $\widetilde{A} \succeq \widetilde{B}$ means $\Re(\widetilde{A}) \geq \Re(\widetilde{B})$ and $\widetilde{A} \preceq \widetilde{B}$ means $\Re(\widetilde{A}) \leq \Re(\widetilde{B})$, and hence $\Re(\widetilde{A})=\Re(\widetilde{B})$. Thus $\widetilde{A} \sim \widetilde{\widetilde{B}}$.

Proposition 4.5. For an arbitrary subset $S$ of $F_{2}$ and $\widetilde{A}, \widetilde{B}, \widetilde{C} \in S, \widetilde{A} \succeq \widetilde{B}$ and $\widetilde{B} \succeq \widetilde{C}$ by $\Re$ on $S$, then $\widetilde{A} \sim \widetilde{C}$ by $\Re$ on $S$.

Proof. If $\widetilde{A} \succeq \widetilde{B}$, then $\Re(\widetilde{A}) \geq \Re(\widetilde{B})$ and if $\widetilde{B} \succeq \widetilde{C}$, then $\Re(\widetilde{B}) \geq \Re(\widetilde{C})$. Since Ranking of triangular type-2 fuzzy set is crisp number, that is, the ranking function mapping each type-2 fuzzy set into a real line, therefore $\Re(\widetilde{A}) \geq \Re(\widetilde{C})$ hence $\widetilde{A} \succeq \widetilde{C}$.

Proposition 4.6. If $\widetilde{A} \cap \widetilde{B}=\emptyset$ and $\widetilde{A}$ is on the right of $\widetilde{B}$, then $\widetilde{A} \succeq \widetilde{B}$.

Proof. If $\widetilde{A} \cap \widetilde{B}=\emptyset$ and $\widetilde{A}$ is on the right of $\widetilde{B}$, then $\Re(\widetilde{A}) \succeq \Re(\widetilde{B})$, that is, $\widetilde{A} \succeq \widetilde{B}$

Proposition 4.7. Let $S$ and $S^{\prime}$ be two arbitrary finite sets of triangular type-2 fuzzy sets in which proposed ranking function can be applied, and $\widetilde{A}, \widetilde{B} \in$ $S \cap S^{\prime}$. Then we obtain the ranking order $\widetilde{A} \succeq \widetilde{B}$ by $\Re$ in $S$ if and only if $\widetilde{A} \succeq \widetilde{B}$ by $\Re$ in $S^{\prime}$.

Proof.

$$
\begin{aligned}
& \text { Given that } \widetilde{A} \succeq \widetilde{B} \text { in } S^{\prime} \\
& \Leftrightarrow \Re(\widetilde{A}) \succeq \Re(\widetilde{B}) \text { in } S^{\prime} \\
& \Leftrightarrow \Re(\widetilde{A}) \succeq \Re(\widetilde{B}) \text { in } S \cap S^{\prime} \quad\left(\because \widetilde{A}, \widetilde{B} \in S \cap S^{\prime}\right) \\
& \Leftrightarrow \Re(\widetilde{A}) \succeq \Re(\widetilde{B}) \text { in } S \quad\left(\because S \cap S^{\prime} \subset S^{\prime}\right) \\
& \Leftrightarrow \widetilde{A} \succeq \widetilde{B} \text { in } S .
\end{aligned}
$$


From the above discussion it is clear that the proposed ranking function satisfies the more reasonable properties than the existing ranking methods. Comparison with existing methods is shown in Table 1.

Table 1. Fulfillment of the axioms for the ordering approaches

\begin{tabular}{|c|c|c|c|c|c|c|c|}
\hline Index & $(P 1)$ & $(P 2)$ & $(P 3)$ & $(P 4)$ & $(P 5)$ & $(P 6)$ & $(P 7)$ \\
\hline Mitchell's method [22] & $\mathrm{N}$ & $\mathrm{N}$ & $\mathrm{N}$ & $\mathrm{Y}$ & $\mathrm{N}$ & $\mathrm{N}$ & $\mathrm{N}$ \\
Wu and Mendel [33] & $\mathrm{Y}$ & $\mathrm{Y}$ & $\mathrm{Y}$ & $\mathrm{Y}$ & $\mathrm{N}$ & $\mathrm{N}$ & $\mathrm{N}$ \\
Proposed Approach & $\mathrm{Y}$ & $\mathrm{Y}$ & $\mathrm{Y}$ & $\mathrm{Y}$ & $\mathrm{Y}$ & $\mathrm{N}$ & $\mathrm{N}$ \\
\hline
\end{tabular}

\section{Multicriteria Decision-Making Problem Based on the Proposed Ranking Approach}

Let $\widetilde{A}=\left\{\widetilde{A}_{1}, \widetilde{A}_{2}, \ldots, \widetilde{A}_{n}\right\}$ be a set of alternatives and let $\widetilde{C}=\left\{\widetilde{C}_{1}, \widetilde{C}_{2}, \ldots, \widetilde{C}_{n}\right\}$ be set of criteria. The values of an alternative on criteria $\widetilde{C}_{j}(j=1,2, \ldots, n)$ are type-2 fuzzy sets $S_{i j}$, which indicates the degree that the alternative $\widetilde{A}_{i}$ satisfies or does not satisfy the criterion $\widetilde{C}_{j}$ given by decision makers or experts according to linguistic values of type-2 fuzzy sets for linguistic terms.

The weights of criterion $\widetilde{C}_{j}(j=1,2, \ldots, n)$ are represented by type- 2 fuzzy sets. The ranking weight value $w_{j}$ for type- 2 fuzzy set is obtained by Eq.(3.6). The normalized weights are obtained using the following equation

$$
w_{j}=\frac{\Re\left(w_{j}\right)}{\sum_{j=1}^{n} \Re\left(w_{j}\right)} .
$$

Therefore, the weighted ranking value for an alternative $\widetilde{A}_{i}(i=1,2, \ldots, m)$ is given by

$$
\Re_{w_{j}}\left(\widetilde{A}_{i}\right)=\sum_{j=1}^{n} w_{j} \Re\left(S_{i j}\right) .
$$

Thus, the calculated weighted ranking value for an alternative is used to rank alternatives and then to select the best one in all the alternatives.

The above method can be summarized as follows:

(1) Calculate the ranking weight value $w_{j}$ for criterion $\widetilde{C}_{j}(j=1,2, \ldots, n)$ by using Eqs.(3.6) and (5.1).

(2) Calculate the weighted ranking value for alternative $\widetilde{A}_{i}(i=1,2, \ldots, m)$ by using Eqs.(3.6) and (5.2).

(3) Rank the alternative and select the best one in accordance with weighted ranking values $\Re_{w_{j}}\left(\widetilde{A}_{i}\right)$. 


\subsection{A Illustrative Example}

A numerical example has been taken to show the applicability of ranking function in multicriteria decision making problem.

Suppose that there is a panel with three alternative to invest the money:

(i) $\widetilde{A}_{1}$ is car company,

(ii) $\widetilde{A}_{2}$ is food company,

(iii) $\widetilde{A}_{3}$ is computer company.

The investment must take a decision according to the following three criteria:

(i) $\widetilde{C}_{1}$ is the risk analysis,

(ii) $\widetilde{C}_{2}$ is the growth analysis,

(iii) $\widetilde{C}_{1}$ is the environmental impact analysis.

The three possible alternatives are to be evaluated under the above three criteria by corresponding to linguistic values of type- 2 fuzzy sets for linguistic terms, as shown in Table 2.

Table 2: Linguistic vales of type-2 fuzzy sets for linguistic terms

\begin{tabular}{|l|c|c|}
\hline \multirow{2}{*}{ linguistic terms } & \multicolumn{2}{|c|}{ Linguistic values of type-2 fuzzy sets } \\
\cline { 2 - 3 } & $\begin{array}{c}\text { Secondary membership function } \\
\text { (SMF) }\end{array}$ & $\begin{array}{c}\text { Primary membership function } \\
\text { (PMF) }\end{array}$ \\
\hline Absolute value & $(0,0,0 ; 0)$ & $(0,0,0 ; 0)$ \\
\hline Low & $(0,0.1,0.2 ; 0.1)$ & $(0,0.1,0.2 ; 0.1)$ \\
\hline Fairly low & $(0.1,0.2,0.4 ; 0.2)$ & $(0.2,0.3,0.4 ; 0.3)$ \\
\hline Medium & $(0.3,0.5,0.7 ; 0.4)$ & $(0.4,0.5,0.6 ; 0.5)$ \\
\hline High & $(0.4,0.6,0.8 ; 0.5)$ & $(0.5,0.6,0.7 ; 0.7)$ \\
\hline Absolutely high & $(0.7,0.8,0.9 ; 0.9)$ & $(0.7,0.8,0.9,0.9)$ \\
\hline
\end{tabular}

Suppose that we called three experts $(k=3)$ to make the decision. They give the linguistic values of type-2 fuzzy sets. The values of alternatives and criteria weights based on the decision makers or experts knowledge are shown in Table 3.

Table 3: Values of alternatives and criteria wights based on the decision makers or experts knowledge 


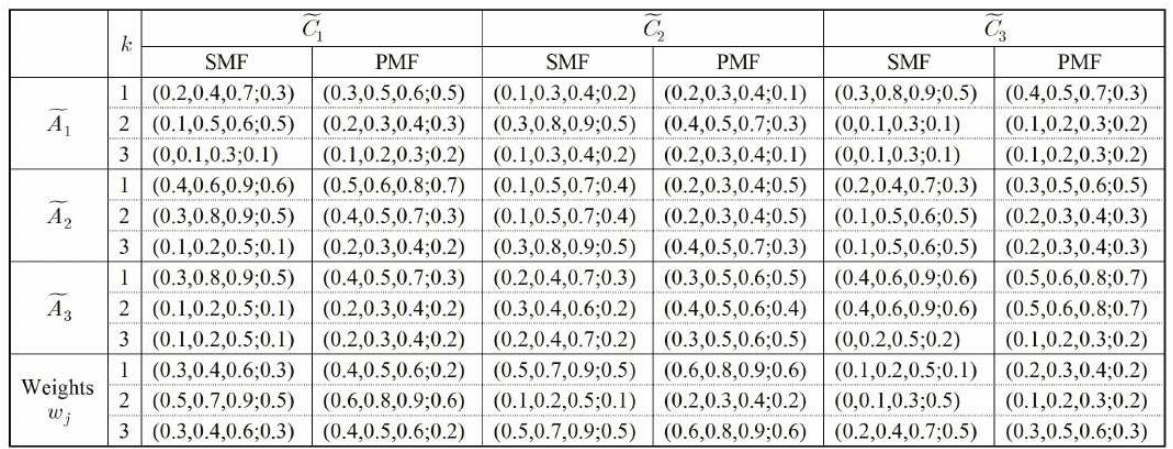

Use the following steps to find the best alternative.

(i) Using Eqs.(3.6) and (5.1), we get the ranking weight value $w_{j}$ for criterion $\widetilde{C}_{j}(j=1,2,3), w_{1}=0.175, w_{2}=0.39$ and $w_{3}=0.435$.

(ii) Using Eqs.(3.6) and (5.2), we get the weighted ranking value for alternative $\widetilde{A}_{i}(i=1,2,3), \Re_{w_{1}}\left(\widetilde{A}_{1}\right)=0.470, \Re_{w_{2}}\left(\widetilde{A}_{2}\right)=1.26$ and $\Re_{w_{3}}\left(\widetilde{A}_{3}\right)=1.38$.

(iii) Rank the alternative as follows: $\widetilde{A}_{1} \prec\left(\widetilde{A}_{2}\right) \prec \widetilde{A}_{3}$.

Thus according to above results the most desirable alternative is $\widetilde{A}_{3}$.

\section{Conclusions}

In this paper, we proposed a innovative method for ordering triangular type-2 fuzzy sets. In the proposed approach we considered both centroid of secondary membership function and footprints of uncertainty as a union of primary membership function. It is shown that the proposed method satisfies five reasonable properties for the ordering of fuzzy quantities, hence it is more reasonable than the existing ranking method. [22, 33]. Some properties of expectation value of the centroid and FOU of triangular type-2 fuzzy sets are discussed. Finally, the proposed ranking approach is applied to multicriteria decision making problem to find the best alternative for different criteria.

\section{References}

[1] S.J. Chen, S.M. Chen, Fuzzy risk analysis based on the ranking of generalized trapezoidal fuzzy numbers, Appl. Intelligence, 26 (2007), 1-11. doi: 10.1007/s10489-006-0003-5

[2] S.M. Chen, J.H. Chen, Fuzzy risk analysis based on ranking generalized fuzzy numbers with dierent heights and dierent spreads, Expert Syst. Appl., 36 (2009), 6833-6842. doi: 10.1016/j.eswa.2008.08.015 
[3] S.M. Chen, K. Sanguansat, Analyzing fuzzy risk based on a new fuzzy ranking method between generalized fuzzy numbers, Expert Syst. Appl., 38 (2011), 2163-2171. doi: 10.1016/j.eswa.2010.08.002

[4] S.M. Chen, M.W. Yang, L.W. Lee, S.W. Yang, Fuzzy multiple attributes group decisionmaking based on ranking interval type-2 fuzzy sets, Expert Syst. Appl., 39 (2012), 52955308. doi: $10.1016 /$ j.eswa.2011.11.008

[5] C.H. Cheng, A new approach for ranking fuzzy numbers by distance method, Fuzzy Sets and Systems, 95 (1998), 307-317. doi: 10.1016/S0165-0114(96)00272-2

[6] D. Dubois, H. Prade, Fuzzy Sets and Systems: Theory and Applications, Academic Press, 1980 .

[7] A. Gupta, A. Kumar, M.K. Sharma, Application of tabu search for solving the biobjective warehouse problem in a fuzzy environment, Iran. J. Fuzzy Syst., 9 (2012), $1-19$.

[8] E. Hisdal, The If THEN ELSE statement and interval-valued fuzzy sets of higher type, Internat. J. Man-Mach. Stud., 15 (1981), 385-455. doi: 10.1016/S0020-7373(81)80051-X

[9] R. Jain, Decision-making in the presence of fuzzy variables, IEEE Trans. Systems Man Cybernet., 6 (1976), 698-703. doi: 10.1109/TSMC.1976.4309421

[10] R.I. John, Type 2 fuzzy sets: an appraisal of theory and applications, Internat. J. Uncertain. Fuzziness Knowledge-Based Systems, 6 (1998), 563-576. doi: $10.1142 / \mathrm{S} 0218488598000434$

[11] R.I. John, Embedded interval valued type-2 fuzzy sets, Proc. 2002 IEEE Int. Conf. Fuzzy Systems, 2 (2002), 1316-1320.

[12] R.I. John, C. Czarnecki, A type-2 adaptive fuzzy inferencing system, Proc. 1998 Int. Conf. Systems, Man Cybernet., (1998) 2068-2073.

[13] A. Kumar, P. Singh, A. Kaur, P. Kaur, RM approach for ranking of generalized trapezoidal fuzzy numbers. Fuzzy Inf. Engin., 1 (2010), 37-47. doi: 10.1007/s12543-010-0036-7

[14] A. Kumar, P. Singh, A. Kaur, P. Kaur, RM approach for ranking of $L-R$ type generalized fuzzy numbers, Soft Computing, 15 (2011), 1373-1381. doi: 10.1007/s00500-010-0676-x

[15] A. Kumar, P. Singh, A. Kaur, P. Kaur, A new approach for ranking nonnormal p-norm trapezoidal fuzzy numbers, Comput. Math. Appl., 61 (2011), 881-887. doi: 10.1016/j.camwa.2010.12.036

[16] A. Kumar, P. Singh, P. Kaur, A. Kaur, A new approach for ranking of $L-R$ type generalized fuzzy numbers, Expert Syst. Appl., 38 (2011), 10906-10910. doi: 10.1016/j.eswa.2011.02.131

[17] H.C. Kwang, J.H. Lee, A method for ranking fuzzy numbers and its application to decision-making. IEEE Trans. Fuzzy Systems, 7 (1999), 677-685. doi: 10.1109/91.811235

[18] T.S. Liou, M.J. Wang, Ranking fuzzy numbers with integral value, Fuzzy Sets and Systems, 50 (1992) 247-255. doi: 10.1016/0165-0114(92)90223-Q

[19] J.M. Mendel, Uncertainty, fuzzy logic and signal processing, Signal Process, 80 (2000), 913-933. doi: 10.1016/S0165-1684(00)00011-6

[20] J.M, Mendel, R.I.B. John, Type-2 fuzzy sets made simple, IEEE Trans. Fuzzy Systems, 10 (2002), 117-127. doi: 10.1109/91.995115 
[21] J.M. Mendel, H. Wu, New results about the centroid of an interval type-2 fuzzy set, including the centroid of a fuzzy granule, Inform. Sci., 177 (2007), 360-377. doi: 10.1016/j.ins.2006.03.003

[22] H.B. Mitchell, Ranking type-2 fuzzy numbers, IEEE Trans. Fuzzy Systems, 14 (2006), 287-294. doi: 10.1109/TFUZZ.2005.864078

[23] M. Mizumoto, K. Tanaka, Some properties of fuzzy sets of type-2, Information and Control, 31 (1976), 312-340. doi: 10.1016/S0019-9958(76)80011-3

[24] M. Mizumoto, K. Tanaka, Fuzzy sets of type-2 under algebraic product and algebraic sum, Fuzzy Sets and Systems, 5 (1976), 277-290. doi: 10.1016/0165-0114(81)90056-7

[25] M. Modarres, S. Sadi-Nezhad, Ranking fuzzy numbers by preference ratio, Fuzzy Sets and Systems, 118 (2001), 429-436. doi: 10.1016/S0165-0114(98)00427-8

[26] N. Ramli, D. Mohamad, A comparative analysis of centroid methods in ranking fuzzy numbers, Eur. J. Sci. Res., 28 (2009), 492-501.

[27] P. Singh, A new approach for the ranking of fuzzy sets with different heights, J. Appl. Res. Technol., 10 (2012), 941-949.

[28] P. Singh, A novel method for ranking generalized fuzzy numbers, J. Inform. Sci. Engin., 31 (2015), 1373-1385.

[29] I.B. Turksen, Interval valued fuzzy sets based on normal forms, Fuzzy Sets and Systems, 20 (1986), 191-210. doi: 10.1016/0165-0114(86)90077-1

[30] I.B. Turksen, Type-2 representation and reasoning for CWW, Fuzzy Sets and Systems, 127 (2002), 17-36. doi: 10.1016/S0165-0114(01)00150-6

[31] X. Wang, E.E. Kerre, Reasonable properties for the ordering of fuzzy quantities (I), Fuzzy Sets and Systems, 118 (2001), 375-385. doi: 10.1016/S0165-0114(99)00062-7

[32] D. Wu, J.M. Mendel, Uncertainty measures for interval type-2 fuzzy sets, Inform. Sci., 177 (2007), 5378-5393. doi: 10.1016/j.ins.2007.07.012

[33] D. Wu, J.M. Mendel, A comparative study of ranking methods, similarity measures and uncertainty measures for interval type-2 fuzzy sets, Inform. Sci., 179 (2009), 1169-1192. doi: $10.1016 /$ j.ins.2008.12.010

[34] R.R. Yager, Fuzzy subsets of type II in decisions, J. Cybernet., 10 (1980), 137-159. doi: $10.1080 / 01969728008927629$

[35] L.A. Zadeh, Fuzzy sets, Information and Control, 8 (1965) 338-353. 
